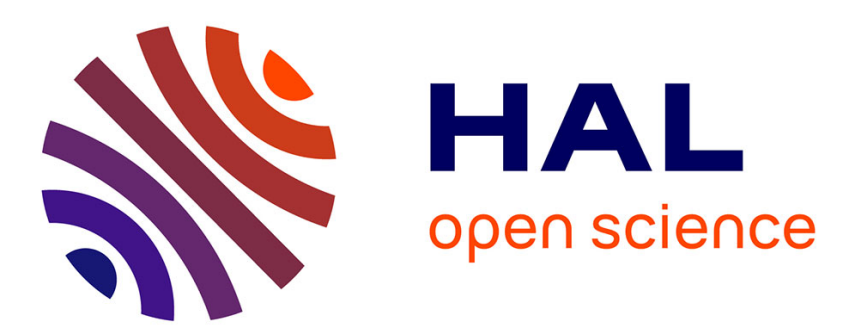

\title{
A Perturbed Inverse Gaussian Process Model with Time Varying Variance-To-Mean Ratio
}

\author{
Songhua Hao, Jun Yang, Christophe Bérenguer
}

\section{To cite this version:}

Songhua Hao, Jun Yang, Christophe Bérenguer. A Perturbed Inverse Gaussian Process Model with Time Varying Variance-To-Mean Ratio. ESREL 2019 - 29th European Safety and Reliability Conference, Sep 2019, Hannover, Germany. pp.739-745, 10.3850/978-981-11-2724-3_0144-cd . hal02324834

\section{HAL Id: hal-02324834 \\ https://hal.science/hal-02324834}

Submitted on 22 Oct 2019

HAL is a multi-disciplinary open access archive for the deposit and dissemination of scientific research documents, whether they are published or not. The documents may come from teaching and research institutions in France or abroad, or from public or private research centers.
L'archive ouverte pluridisciplinaire HAL, est destinée au dépôt et à la diffusion de documents scientifiques de niveau recherche, publiés ou non, émanant des établissements d'enseignement et de recherche français ou étrangers, des laboratoires publics ou privés. 


\title{
A PERTURBED INVERSE GAUSSIAN PROCESS MODEL WITH TIME VARYING VARIANCE-TO-MEAN RATIO
}

\author{
SONGHUA HAO \\ School of Reliability and Systems Engineering, Beihang University, Beijing, China. \\ E-mail: haosonghua@buaa.edu.cn \\ JUN YANG \\ School of Reliability and Systems Engineering, Beihang University, Beijing, China. \\ E-mail:tomyj2001@buaa.edu.cn \\ CHRISTOPHE BERENGUER \\ Univ. Grenoble Alpes, CNRS, Grenoble INP, GIPSA-lab, Grenoble, France. \\ E-mail: Christophe.Berenguer@grenoble-inp.fr
}

\begin{abstract}
The inverse gaussian (IG) process has become a common model for reliability analysis of monotonic degradation processes. The traditional IG process model assumes that the degradation increment follows an IG distribution, and the variance-to-mean ratio (VMR) is constant with time. However, for the degradation paths of some practical applications, e.g., the GaAs laser degradation data that motivated to propose the IG process, the VMR is actually time varying. Confronted with this, we propose an IG process model with measurement errors that depend on the actual degradation level. According to different forms or parameter values of the dependence function, the VMR of the degradation paths can display different time varying patterns. The maximum likelihood estimation method is developed in a step-by-step way, combined with numerical integration method and heuristic optimization method. Finally, the GaAs laser example is revisited to illustrate the effectiveness of the proposed model, which indicates that the introduction of statistically dependent measurement error can provide better fitting results.
\end{abstract}

Keywords: Inverse gaussian process, Measurement error, Time varying VMR, GaAs laser.

\section{Introduction}

Considering the increasing difficulty of obtaining sufficient failure data in normal reliability tests, degradation analysis has been developed as a popular way for reliability assessment. Among many well-developed stochastic degradation models, the inverse gaussian (IG) process has attracted much attention for modelling monotonic degradation processes. For the GaAs laser degradation data in (Meeker and Escobar 1998), the Wiener and Gamma processes showed bad fitting results, and an IG process model was firstly proposed as a good alternative (Wang and $\mathrm{Xu}$ 2010). Then Ye and Chen studied its meaningful physical interpretation, and also pointed out its inverse relation with Wiener process (Ye and Chen 2014). These pioneering works led to extensive further research of the IG process, such as the remaining useful life estimation (Pan, Liu, and Cao 2016), accelerated degradation test planning (Ye et al. 2014), condition-based maintenance optimization (Chen et al. 2015) and bivariate degradation analysis (Peng et al. 2016).

Edited by Michael Beer and Enrico Zio

Copyright (C2019 by ESREL2019 Organizers. Published by Research Publishing, Singapore

ISBN: 981-973-0000-00-0 :: doi: 10.3850/981-973-0000-00-0 esrel2019-paper

The traditional IG process model assumes that the degradation increments follow an IG distribution. To keep the additivity of the IG distributions and the mathematical tractability of the traditional IG process model, the variance-tomean ratio (VMR) of the degradation model is constant with time. However, for the degradation paths of some practical applications, the VMR may be time varying (Guida, Postiglione, and Pulcini 2012). Therefore, in order to further improve the fitting performance of the IG process model, it is meaningful to extend it by considering time varying VMR.

Due to various sources of uncertainty in measurement processes, the measured degradation may not always represent perfectly the true degradation, and this greatly affects the accuracy of model parameter estimation and reliability assessment. To the best of our knowledge, existing researches about IG process model with measurement error are still limited. To model measurement errors, a common approach is to introduce a normal distributed variable with mean zero and constant standard 


\section{SONGHUA HAO, JUN YANG and CHRISTOPHE BERENGUER}

deviation. (Ye and Xie 2015) made a good review of stochastic degradation process models contaminated by random white noises.

However, from the viewpoints of measurement systems analysis (Rabinovich 2005, 2013), sometimes it is more practical to assume that the measurement error is statistically dependent with the actual but hidden degradation value (Zhai and Ye 2017). Based on this assumption, (Pulcini 2016) developed a novel Gamma process model, where the measurement error is modelled by a zero-mean normally distributed variable, and its standard deviation is dependent on the actual degradation value. With different dependent function forms and different parameter values, the VMR of the degradation model shows completely different time varying patterns. With the same assumption of statistically dependent measurement error, (Giorgio, Mele, and Pulcini 2018) replaced the normal distributed variable by a three-parameter inverse gamma variable, which improved the degradation model by excluding non-negative degradation measurements.

Motivated by the GaAs laser example and similar research for Gamma process model, we propose a new perturbed IG process model, where the standard deviation of the normally distributed measurement error depends on the actual degradation level. Descriptions of the new model are presented in Section 2, with the derivation of time varying VMR. Section 3 provides the maximum likelihood estimation method in a step-by-step way. Section 4 revisits the GaAs laser example to illustrate the effectiveness of the proposed model. Some conclusions are given in Section 5.

\section{The perturbed inverse gaussian process model}

The traditional IG process model, denoted by $\{X(t), t \geq 0\}$, is defined to have the following properties:

(1) $X(0)=0$ with probability 1 ;

(2) The degradation increments at disjoint time intervals are independent with each other, i.e., for any $t_{4}>t_{3} \geq t_{2}>t_{1} \geq 0, X\left(t_{2}\right)-X\left(t_{1}\right)$ is independent with $X\left(t_{4}\right)-X\left(t_{3}\right)$;
(3) The degradation increment is assumed to be IG distributed, i.e., for any $t>s \geq 0$, $X(t)-X(s) \sim I G\left(\beta \Delta \Lambda(t), \eta \Delta \Lambda^{2}(t)\right)$, where $\beta$ and $\eta$ are respectively the slope and shape parameter, and $\Lambda(t)$ is the nonlinear drift function.

According to these definitions, we can know that the degradation performance $X(t)$ follows an IG distribution $I G\left(\beta \Lambda(t), \eta \Lambda^{2}(t)\right)$. And its probability density function (PDF) is as follows:

$$
f_{X}(x)=\sqrt{\frac{\eta \Lambda^{2}(t)}{2 \pi x^{3}}} \exp \left\{-\frac{\eta[x-\beta \Lambda(t)]^{2}}{2 \beta^{2} x}\right\}(1)
$$

Furthermore, it can be derived that the expectation, variance and VMR of $X(t)$ are respectively:

$$
\begin{gathered}
E[X(t)]=\beta \Lambda(t) \\
\operatorname{Var}[X(t)]=\frac{[\beta \Lambda(t)]^{3}}{\eta \Lambda^{2}(t)}=\frac{\beta^{3}}{\eta} \Lambda(t) \\
\operatorname{VMR}[X(t)]=\frac{\operatorname{Var}[X(t)]}{E[X(t)]}=\frac{\beta^{2}}{\eta}
\end{gathered}
$$

It can be indicated that although the expectation and variance of the IG process are both time varying, the VMR of $X(t)$ is eventually constant, and does not change over time. This theoretical result is not fully consistent with the real observation in some practical applications, so we propose a novel perturbed IG process model, based on the actual degradation process and measurement error:

$$
Y_{k}=X_{k}+\varepsilon_{k}
$$

where $Y_{k}=Y\left(t_{k}\right), X_{k}=X\left(t_{k}\right), \varepsilon_{k}=\varepsilon\left(t_{k}\right)$ are respectively the measured degradation, actual degradation, measurement error at the $k$ th measurement time point $t_{k}$, either in constant inspection interval or non-constant one. 
Most previous studies have assumed that $X_{k}$ and $\varepsilon_{k}$ are independent, and in this case, the VMR of the measured degradation process decreases over time. To account for more timevarying patterns of the VMR, we propose to assume that the measurement error is statistically dependent on the actual degradation performance. Here we let $\varepsilon_{k}$ be normally distributed with zero mean, whose standard deviation $\sigma_{\varepsilon_{k}}\left(x_{k}\right)$ is a non-decreasing dependence function of the temporal actual degradation performance. Therefore, its conditional PDF can be written as follows:

$$
f_{\varepsilon_{k} \mid X_{k}}\left(z \mid x_{k}\right)=\frac{1}{\sqrt{2 \pi} \sigma_{\varepsilon_{k}}\left(x_{k}\right)} e^{-\frac{z^{2}}{2 \sigma_{\varepsilon_{k}}^{2}\left(x_{k}\right)}}
$$

Theorem 1. For the perturbed IG process model with statistically dependent measurement error, the VMR is derived to be:

$$
\begin{aligned}
& \operatorname{VMR}\left(Y_{k}\right) \\
& =\frac{\beta^{3}}{\eta}+\frac{a^{2} \sqrt{\eta}}{\sqrt{2 \pi}} \int_{0}^{+\infty} x^{2 b-3} e^{-\frac{\eta\left[x-\beta \Lambda\left(t_{k}\right)\right]^{2}}{2 \beta^{2} x}} d x
\end{aligned}
$$

Proof. According to Eqs. (5) and (6), the PDF of $Y_{k}$ on condition of $X_{k}$ is as follows:

$$
\begin{aligned}
& f_{Y_{k} \mid X_{k}}\left(y \mid x_{k}\right)=f_{\varepsilon_{k}}\left(y-x_{k} \mid x_{k}\right) \\
& =\frac{1}{\sqrt{2 \pi} \sigma_{\varepsilon_{k}}\left(x_{k}\right)} \exp \left\{-\frac{\left(y-x_{k}\right)^{2}}{2 \sigma_{\varepsilon_{k}}^{2}\left(x_{k}\right)}\right\}
\end{aligned}
$$

And the conditional expectation and variance is respectively:

$$
\begin{gathered}
E\left[Y_{k} \mid x_{k}\right]=x_{k} \\
\operatorname{Var}\left(Y_{k} \mid x_{k}\right)=\sigma_{\varepsilon_{k}}^{2}\left(x_{k}\right)
\end{gathered}
$$

The unconditional expectation and variance are:

$$
E\left[Y_{k}\right]=E_{X}\left[E_{Y}\left(Y_{k} \mid x_{k}\right)\right]=\beta \Lambda\left(t_{k}\right)
$$

$$
\begin{aligned}
& \operatorname{Var}\left(Y_{k}\right) \\
& =E_{X}\left[\operatorname{Var}\left(Y_{k} \mid x_{k}\right)\right]+\operatorname{Var}_{X}\left[E\left(Y_{k} \mid x_{k}\right)\right] \\
& =E_{X}\left[a^{2} x_{k}^{2 b}\right]+\frac{\beta^{3}}{\eta} \Lambda\left(t_{k}\right) \\
& =\frac{\beta^{3}}{\eta} \Lambda\left(t_{k}\right)+\frac{a^{2} \sqrt{\eta}}{\sqrt{2 \pi}} \Lambda\left(t_{k}\right) \int_{0}^{+\infty} x^{2 b-3} e^{-\frac{\eta\left[x-\beta \Lambda\left(t_{k}\right)\right]^{2}}{2 \beta^{2} x}} d x
\end{aligned}
$$

Therefore, through dividing Eq. (11) by Eq. (12), we can obtain the VMR of the proposed model, and this completes the proof of Theorem 1 .

In the rest of this paper, it is particularly assumed that the standard deviation of measurement error is a power function of the actual degradation performance, i.e., $\sigma_{\varepsilon_{k}}\left(x_{k}\right)=a x_{k}^{b}, a, b>0$. This assumption is due to the flexibility of power function and motivated by (Pulcini 2016), and the perturbed IG process model with this statistical dependent measurement error is denoted by $M_{S D M E}$. Note that this model is rather general and flexible, and have two simpler models as special cases, i.e., the traditional IG process model ( $a=0$, denoted by $\left.M_{T I G}\right)$, and the perturbed IG process model with statistically independent measurement error ( $b=0, a \neq 0$, denoted by $M_{\text {SIME }}$ ).

Additionally, some simulations have been done to show the time-varying patterns of VMR in Fig. 1. We can see that the VMR of the proposed model can display various kinds of trends, depending on different values of $a, b$.

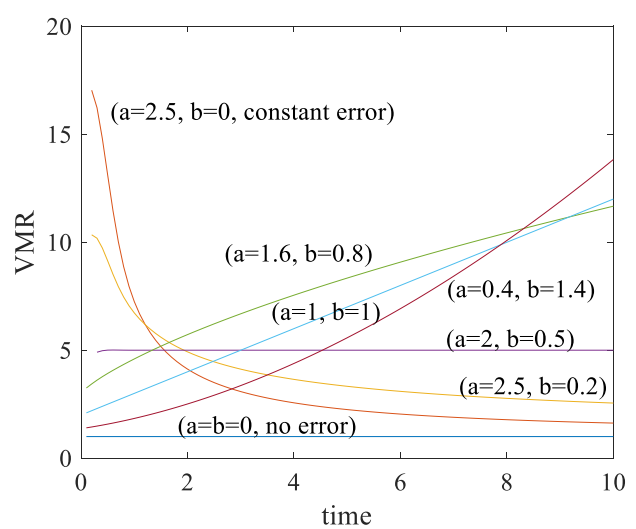

Fig. 1. Time-varying patterns of VMR under different values of $a, b$ 


\section{Parameter estimation}

For the traditional IG process model, the degradation increments of two successive measurements are independent IG distributed variables, and the likelihood function can be easily obtained. However, for the proposed perturbed IG process model, the introduction of measurement errors leads to the fact that the degradation increments are no longer independent. Based on the concept of conditional probability, the likelihood function for all measured samples can be expressed as:

$$
\begin{aligned}
& L(\mathbf{y} ; \Theta)=\prod_{i=1}^{n} f_{Y_{i, 1}}\left(y_{i, 1}\right) f_{Y_{i, 2} \mid Y_{i, 1}}\left(y_{i, 2} \mid y_{i, 1}\right) \\
& \cdots f_{Y_{i, m_{i}} \mid Y_{i, m-1}}\left(y_{i, m_{i}} \mid y_{i, m_{i}-1}\right)
\end{aligned}
$$

where $n$ is the sample size, $t_{i, k}, k=1,2, \cdots, m_{i}$ is the $k$ th measurement time for sample $i$, $y_{i, k}=y\left(t_{i, k}\right)$ is the measured degradation performance for sample $i$ at time $t_{i, k}$, and $f_{Y_{i, k} \mid Y_{i, k-1}}\left(y_{i, k} \mid y_{i, k-1}\right), k=2,3, \cdots, m_{i}$ is the PDF of $Y_{i, k}$ conditioned on $Y_{i, k-1}$.

To obtain the above likelihood function, it is required to derive the conditional PDF $f_{Y_{i, k} \mid Y_{i, k-1}}\left(y_{i, k} \mid y_{i, k-1}\right)$, and this can be computed in the following step-by-step way, combined with the numerical integration method for the complex integrations.

1) Derive the PDF of the actual degradation $X_{i, k-1}$ on condition of the measured degradation $Y_{i, k-1}$, both at time $t_{i, k-1}$ :

$$
\begin{aligned}
& f_{X_{i, k-1} \mid Y_{i, k-1}}\left(v \mid y_{i, k-1}\right) \\
= & \frac{f_{Y_{i, k-1} \mid X_{i, k-1}}\left(y_{i, k-1} \mid v\right) \cdot f_{X_{i, k-1}}(v)}{f_{Y_{i, k-1}}\left(y_{i, k-1}\right)} \\
= & \frac{f_{Y_{i, k-1} \mid X_{i, k-1}}\left(y_{i, k-1} \mid v\right) \cdot f_{X_{i, k-1}}(v)}{\int_{0}^{+\infty} f_{Y_{i, k-1} \mid X_{i, k-1}}\left(y_{i, k-1} \mid u\right) f_{X_{i, k-1}}(u) d u}
\end{aligned}
$$

where $f_{Y_{i, k-1} \mid X_{i, k-1}}\left(y_{i, k-1} \mid u\right)$ and $f_{X_{i, k-1}}(u)$ can be referred to Eqs. (8) and (1), respectively.

2) Based on Eq. (14), we can update and obtain the PDF of the actual degradation $X_{i, k}$ on condition of the measured degradation $Y_{i, k-1}$, respectively at times $t_{i, k}$ and $t_{i, k-1}$ :

$$
\begin{aligned}
& f_{X_{i, k} \mid Y_{i, k-1}}\left(w \mid y_{i, k-1}\right) \\
& =\int_{0}^{+\infty} f_{X_{i, k} \mid X_{i, k-1}}(w \mid v) f_{X_{i, k-1} \mid Y_{i, k-1}}\left(v \mid y_{i, k-1}\right) d v
\end{aligned}
$$

where $\quad f_{X_{i, k} \mid X_{i, k-1}}(w \mid v)=f_{X_{i, k}-X_{i, k-1}}(v-w)$ can also be referred to the PDF of degradation increment in Eq. (1).

3) Finally, the conditional PDF of the measured degradation at two successive times can be expressed as:

$$
\begin{aligned}
& f_{Y_{i, k} \mid Y_{i, k-1}}\left(y_{i, k} \mid y_{i, k-1}\right) \\
& =\int_{0}^{+\infty} f_{Y_{i, k} \mid X_{i, k}}\left(y_{i, k} \mid w\right) f_{X_{i, k} \mid Y_{i, k-1}}\left(w \mid y_{i, k-1}\right) d w
\end{aligned}
$$

where $f_{Y_{i, k} \mid X_{i, k}}\left(y_{i, k} \mid w\right)$ can be referred to Eq. (8).

Therefore, the likelihood function for all measured samples can be given by plugging Eq. (16) into (13). And by maximizing the likelihood function (or the logarithm value), a heuristic optimization method, such as a genetic algorithm, can be used to estimate the model parameters.

\section{Illustrative example}

In this Section, we reanalyse the GaAs laser example that motivated to propose the IG process, and demonstrate the effectiveness of the proposed perturbed IG process model.

Firstly, in order to enlighten the limitation of the traditional IG process model (i.e., constant VMR) for some real data, we plotted the GaAs laser degradation paths and the time varying VMR, respectively in Fig. 2 and Fig. 3. It can be seen that the VMR obviously increases with time. Furthermore, we have fitted the time varying VMR with several candidate, and find that a simple linear function can provide rather 
good fitting results, with the square of the Pearson correlation coefficient to be 0.9955 . Therefore, based on the simulation results in section 2, an initial guess of the model parameter $b$ will be 1 .

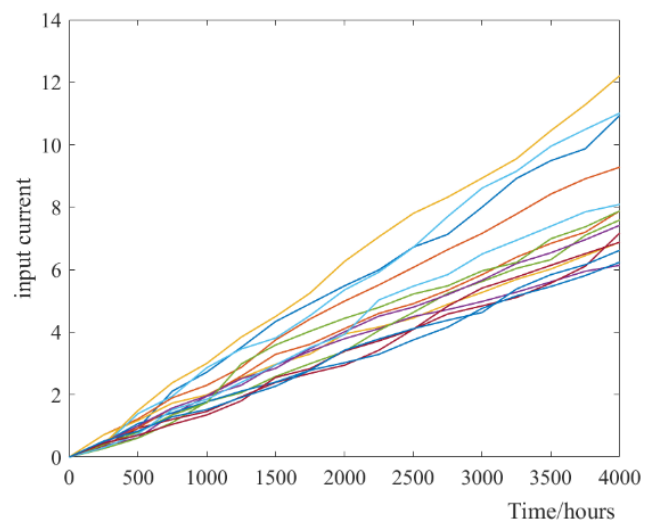

Fig. 2. Degradation paths of the GaAs laser example over time

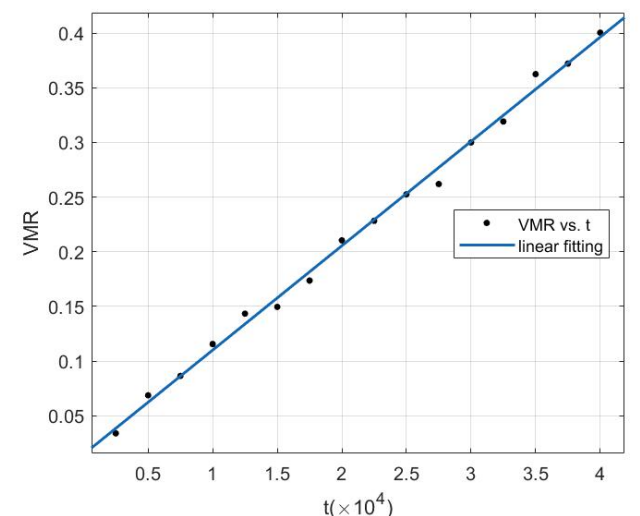

Fig. 3. The VMR of the GaAs laser example over time

Therefore, it is natural to apply the proposed perturbed IG process model to the GaAs laser example. Based on the laser degradation data, we estimate the relevant model parameters through the step-by-step method in Section 3. Furthermore, the following Table 1 lists the comparison results of three candidate models, i.e., $M_{T I G}, M_{S I M E}$ and $M_{S D M E}$ (donoted in Section 2). We can see that compared to the traditional IG process model, model $M_{\text {SIME }}$ provides slightly poorer fitting with smaller loglikelihood value, and this may be because that statistically independent measurement error leads to decreasing VMR, which deviates farther from the VMR of laser data. Besides, the proposed $M_{S D M E}$, with the smallest Akaike information criterion (AIC), performs best for the laser example, which illustrates the significance of introducing statistically dependent measurement error, and also the effectiveness of the proposed perturbed IG process model.

Table 1. Log-likelihoods and AICs of the 3 models for $\mathrm{GaAs}$ laser degradation data.

\begin{tabular}{lll}
\hline Candidate model & Log-likelihood & AIC \\
\hline$M_{\text {TIG }}$ & 75.03 & -146.06 \\
$M_{\text {SIME }}$ & 75.02 & -144.04 \\
$M_{S D M E}$ & 77.69 & -147.38 \\
\hline
\end{tabular}

\section{Conclusion}

The VMR of the traditional IG process model is constant with time. In accordance with the time varying features of many practical degradation processes, we propose a novel perturbed IG process model with statistically dependent measurement error. Particularly, it is assumed that the standard deviation of measurement error is a power law function of the actual degradation performance, and simulation results indicate that the VMR of the proposed model can display various kinds of time varying trends, depending on different values of the dependence function. The parameter estimation is developed in a stepby-step way. And the GaAs laser degradation data, with approximately linear increasing VMR, is reanalysed to indicate the effectiveness of the proposed perturbed IG process model. Compared to the traditional IG process model with constant VMR and the model with independent measurement error and decreasing VMR, the proposed perturbed IG process model performs best to fit the laser example.

For further research of this work, non-gaussian or truncated measurement error can be investigated to model the practical non-negative measurements better. Besides, random effects can be further introduced to represent the unit-tounit variability. Another interesting direction can be remaining useful life estimation and condition-based maintenance based on the perturbed IG process model.

\section{Acknowledgement}

This work was supported by the National Natural Science Foundation of China (71672006), and the 
Fundamental Research Funds for the Central Universities under Grant No. YWF-18-BJ-J-72.

\section{References}

Chen, Nan, Zhi-Sheng Ye, Yisha Xiang, and Linmiao Zhang (2015). Condition-Based Maintenance Using the Inverse Gaussian Degradation Model. European Journal of Operational Research 243 (1): 190-199.

Giorgio, Massimiliano, Agostino Mele, and Gianpaolo Pulcini (2018). A Perturbed Gamma Degradation Process with Degradation Dependent NonGaussian Measurement Errors. Applied Stochastic Models in Business and Industry, July.

Guida, M., F. Postiglione, and G. Pulcini (2012). A Time-Discrete Extended Gamma Process for Time-Dependent Degradation Phenomena. Reliability Engineering \& System Safety 105 (September): 73-79.

Meeker, William Q, and Luis Escobar (1998). Statistical Methods for Reliability Data. Wiley.

Pan, Donghui, Jia-Bao Liu, and Jinde Cao (2016). Remaining Useful Life Estimation Using an Inverse Gaussian Degradation Model. Neurocomputing 185 (April): 64-72.

Peng, Weiwen, Yan-Feng Li, Yuan-Jian Yang, ShunPeng Zhu, and Hong-Zhong Huang (2016). Bivariate Analysis of Incomplete Degradation Observations Based on Inverse Gaussian Processes and Copulas. IEEE Transactions on Reliability 65 (2): 624-639.

Pulcini, Gianpaolo (2016). A Perturbed Gamma Process with Statistically Dependent Measurement Errors. Reliability Engineering \& System Safety 152 (August): 296-306.

Rabinovich, Semyon G (2005). Measurement Errors and Uncertainties: Theory and Practice. 3rd ed. New York: Springer-Verlag.

Rabinovich, Semyon G (2013). Evaluating Measurement Accuracy: A Practical Approach. 2nd ed. New York: Springer-Verlag.

Wang, Xiao, and Dihua Xu (2010). An Inverse Gaussian Process Model for Degradation Data. Technometrics 52 (2): 188-197.

Ye, Zhi-Sheng, Liang-Peng Chen, Loon Ching Tang, and Min Xie (2014). Accelerated Degradation Test Planning Using the Inverse Gaussian Process. IEEE Transactions on Reliability 63 (3): 750-763.

Ye, Zhi-Sheng, and Nan Chen (2014). The Inverse Gaussian Process as a Degradation Model. Technometrics 56 (3): 302-311.

Ye, Zhi-Sheng, and Min Xie (2015). Stochastic Modelling and Analysis of Degradation for Highly Reliable Products. Applied Stochastic Models in Business and Industry 31 (1): 16-32.

Zhai, Q., and Z. Ye (2017). Robust Degradation Analysis With Non-Gaussian Measurement Errors. IEEE Transactions on Instrumentation and Measurement 66 (11): 2803-12. 